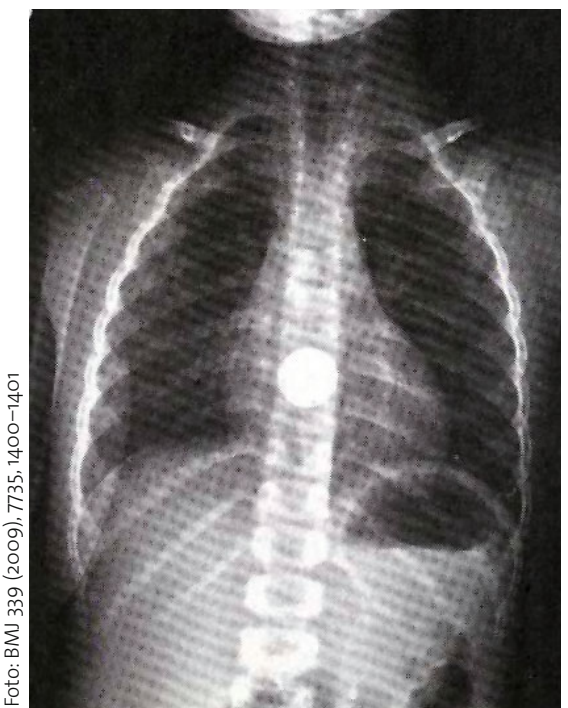

Ein Börsencrash vermindert den kindlichen Appetit auf Münzen nicht.

_ Zwischen August 2006 und Juli 2009 kategorisierte man sämtliche in der Endoskopieabteilung des Massachusetts General Hospital aus dem Ösophagus von Kindern geborgenen $\mathrm{Ob}$ jekte. Der finanzielle Gesamtwert der geborgenen Münzen wurde mit dem durchschnittlichen Monatswert des Dow-Jones-Index korreliert.

\section{Keine Wirtschaftskrise im Kindermagen}

\begin{abstract}
Die Aspiration und Ingestion von Fremdkörpern ist ein bekanntes Problem bei Kleinkindern. Zu den beliebtesten Objekten, die verschluckt werden, gehören Münzen. Nachdem die verschluckten Objekte das sozioökonomische Umfeld des Kleinkindes widerspiegeln, nahmen die Autoren der Studie an, dass die Wirtschaftskrise auch hier ihren Niederschlag gefunden haben könnte.
\end{abstract}

Der Wert Münzen betrug 1,03 \$. Teilte man den Untersuchungszeitraum ein in jeweils vor und nach dem Oktober 2008, so wurden keine signifikanten Veränderungen gefunden. Der durchschnittliche Dow-Jones-Index am Monatsende war in den beiden Zeiträumen jedoch signifikant unterschiedlich (12537 vs. 8388; p < 0,0001)

\section{Kommentar}

Obwohl man an der Validität des Modells Zweifel haben kann, wonach von Kleinkindern verschluckte Objekte die sozioökonomischen Bedingungen widerspiegeln, sehen die Autoren mit dem Studienergebnis ihre Hypothese nicht widerlegt und machen dafür nicht eliminierbare konfundierende Faktoren verantwortlich.
Dazu gehört vor allem die Verweigerung von Krediten in der Krise. Ein restriktives Verhalten der Banken bei der Kreditvergabe hat unweigerlich zur Folge, dass die Verfügbarkeit und der Gebrauch von nicht schluckbaren Kreditkarten zugunsten von eher genießbarem Kleingeld zurückgeht. Diese Veränderung bei den Zahlungsmitteln täuscht durch eine Erhöhung der Anzahl verschluckter Münzen einen Wohlstand vor, der tatsächlich nicht mehr vorhanden ist. Die Autoren haben keinen Lösungsvorschlag, wie man dieses Dilemma umgehen könnte, und schlagen daher auch keine weiteren Studien zu dem Thema vor.

H. S. FüEßL .

- P.G. Firth et al.

Ingested foreign bodies and societal wealth: three years observational study of swallowed coins. BMJ 339 (2009), 7735, 1400-1401

\title{
Haben auch Sie ein Opioid-Verweigerungs-Syndrom (OVS)?
}

Seit Jahrzehnten verbreitet sich eine eigenartige, hoch kontagiöse und in der Regel während des Medizinstudiums übertragene Erkrankung unter Ärzten: das Opioid-Verweigerungs-Syndrom (OVS).

- Das OVS ist eine neuropsychiatrische Erkrankung, die zu schwerer Beeinträchtigung der Berufsfähigkeit führen kann.

Das hoch kontagiöse Syndrom wird in der Regel am Krankenbett von akademischen Lehrern auf Studenten übertragen. Typischerweise stehen diese Ärzte vor allem im Banne einer Diagnose, anstatt sich in erster Linie um das Befinden ihrer Patienten zu kümmern. Jeder Patient, der effektive Schmerzbehandlungen verlangt, ist in ihren Augen ein „Drogenabhängiger“. Vorübergehende Remissionen des OVS werden bei Ärzten beobachtet, die Krankenhausverwalter, Fachanwälte für Kunstfehlerprozesse oder Personen behandeln, die sie an sie selbst erinnern.
Mit vier Fragen lässt sich die Verdachtsdiagnose eines OVS stellen:

(1) Haben Sie von Ihren Kollegen schon einmal verlangt, die Opioidverschreibungen zu reduzieren?

2 Ärgern Sie sich, wenn Patienten behaupten, Opioide würden Ihre Schmerzen lindern?

(3) Haben Sie sich jemals wegen einer Opioidverschreibung schuldig gefühlt?

(4) Vermeiden Sie den Augenkontakt mit Ihren Patienten?

Zwei und mehr positive Antworten machen ein OVS wahrscheinlich. Die Vorschläge zur Behandlung des OVS sind vielfältig, doch dürfte am ehesten eine goldene Lebensregel effektiv sein: Irgendwann werden die meisten Ärzte an sich selbst Schmerzen erfahren, die sie zu einem Kollegen führen. Fragen Sie sich, wie Sie dann gerne behandelt werden würden!

H. S. FüEßL =

\footnotetext{
- B.D. Veysman

(veysmabo@umdnj.edu), Prescriber's narcophobia syndrome: physicians' disease and patients' misfortune. BMJ 339 (2009) 7735, 1422
} 